\title{
Prevalence of autoimmune thyroid disorders in kin/relatives with those diseases
}

M. Carsote ${ }^{1}$, C. D. Staicu ${ }^{2}$, A. Clodeanu ${ }^{2}$, D. Peretianu ${ }^{1}$,

1. "Parhon" Institute of Endocrinology; 2. Medical Center "Povernei "

INTRODUCTION \& AIM

Bucharest, Romania

Thyroid autoimmune diseases associate a familial pattern.

We aim to investigate the prevalence of relationships between kin/relatives with a known thyroid immune disorder related to Hashimoto thyroiditis. MATERIAL \& METHOD

1. Thyroid disease diagnosis: a. TPO Antibodies (ATPO) $>34 \mathrm{u} / \mathrm{ml}=$ Hashimoto thyroiditis (HT); b. ATPO = normal but with high antithyroglobuline $($ ATG $)=$ thyroiditis with only hyper-ATG (T-ATG); c. hypothyroidism without high ATPO/ATG = idiopathic mixedema (IM).

2. Searching for a relationships between relatives (including anamnesis). RESULTS

1401 patients with HT, 129 patients with T-ATG, and 108 Idiopathic myxedema. A specific kin/relationship could be identified in 189 patients. The distribution is tabulated below:

\begin{tabular}{|c|c|c|c|c|c|c|c|}
\hline \multirow[t]{2}{*}{ Familial relationships } & \multicolumn{3}{|c|}{ Pairs } & \multicolumn{3}{|c|}{ Nonregistered - only by declaration } & \multirow{2}{*}{ Total } \\
\hline & HT & TATG & MIX ID & HT & TATG & ID MIX & \\
\hline Mother-Daughter & 74 & 6 & 5 & 19 & 3 & $\mathbf{0}$ & 86 \\
\hline Mother-Son & 12 & 1 & $\mathbf{0}$ & 3 & $\mathbf{0}$ & $\mathbf{0}$ & 13 \\
\hline Father-Daughter & 8 & $\mathbf{0}$ & $\mathbf{0}$ & 3 & $\mathbf{0}$ & $\mathbf{0}$ & 11 \\
\hline Father-Son & 3 & $\mathbf{0}$ & $\mathbf{0}$ & $\mathbf{0}$ & $\mathbf{0}$ & $\mathbf{0}$ & 2 \\
\hline $\begin{array}{l}\text { Grandmother- } \\
\text { Grandson/daughter }\end{array}$ & 1 & $\mathbf{0}$ & $\mathbf{0}$ & 1 & $\mathbf{0}$ & $\mathbf{0}$ & 2 \\
\hline Uncle/Aunt-Nephew & 4 & 1 & $\mathbf{0}$ & 1 & $\mathbf{0}$ & $\mathbf{0}$ & 4 \\
\hline Wife-Husband & 4 & $\mathbf{0}$ & 1 & $\mathbf{0}$ & $\mathbf{0}$ & $\mathbf{0}$ & 5 \\
\hline Sister-Sister & 19 & 2 & 3 & 6 & 1 & 1 & 23 \\
\hline Sister-Brother & 5 & $\mathbf{0}$ & $\mathbf{0}$ & 4 & $\mathbf{0}$ & $\mathbf{0}$ & 8 \\
\hline Total pairs & 130 & 10 & 9 & 37 & 4 & 1 & 154 \\
\hline Multiple members & 5 & $\mathbf{0}$ & $\mathbf{0}$ & & & & 5 \\
\hline Patients (number) & 197 & 16 & 17 & & & & 189 \\
\hline Patients (percent) & 11.28 & 12.40 & 15.74 & & & & 13.17 \\
\hline
\end{tabular}

A relationship between kin was established in $1.28 \%$ of patients with $\mathrm{HT}$, in $12.4 \%$ of subjects with T-ATG and $15.74 \%$ of those with IM.

\section{Conclusion}

Overall, based on our observations, the penetration of a relationship between two kin with Hashimoto thyroiditis and related disorders was registered in $13.7 \%$ of patients. 